\title{
A clinicopathological study of ascites with special reference to malignant etiology in a tertiary care hospital in Kolkata
}

\author{
Ray $D^{1}$, Chakrabarti $S^{2}$, Chakraborty $A^{3}$, Kundu $D^{4}$, Bandyopadhyay $R^{5}$ \\ ${ }^{1}$ Dr Debes Ray, Associate Professor, Department of Biochemistry, Medical College Kolkata, India, ${ }^{2}$ Dr Srabani \\ Chakrabarti, Associate Professor, Department of Pathology, Calcutta National Medical College, Kolkata, India, \\ ${ }^{3}$ Dr Arkaprabha Chakraborty, Junior Resident, Department of Medicine, Medical College Kolkata, India, ${ }^{4}$ Dr Dipankar \\ Kundu, Assistant Professor, Department of Biochemistry, Medical College Kolkata, India, ${ }^{5}$ Dr Ramtanu Bandyopadhyay, \\ Professor, Department of Medicine, Malda Medical College, Malda, West Bengal, India.
}

Address for Correspondence: Dr. Debes Ray, 13/4, Jagannath Ghosh Road, Block- B, P.O. Kasba. Kolkata, West Bengal. India. E- mail:drdebes_ray@yahoo.co.in

\begin{abstract}
Introduction: Ascites is one the most common presenting complaints in tertiary care hospitals. Cirrhosis accounts for majority of cases of ascites. Cardiac ascites, peritoneal carcinomatosis, and "mixed" ascites resulting from cirrhosis and its complications account for rest of the cases. Less common causes of ascites include massive hepatic metastasis, infection (tuberculosis, Chlamydia), pancreatitis, and renal disease (nephrotic syndrome). Hepatitis B virus (HBV) infection, hepatitis $\mathrm{C}$ virus (HCV) infection, and alcoholic liver disease are the main causes of cirrhosis in India and rest of the world. Aim and objective: The aim of the study is to explore the etiopathology of ascites in the patients who were admitted to a tertiary care hospital in eastern India. Materials and methods: A hospital based cross-sectional study is conducted over a period of one year in Medical College and Hospital, Kolkata. 100 patients of ascites above $18 y$ rs of age were studied. Clinico-pathological, hematological and biochemical parameters along with imaging findings were documented and were presented in tabular, graphical and statistical means. Results: In our study the most common causes of ascites found were alcoholism (36\%) followed by malignant ascites (18\%). It was also observed that most of the patients were between 40-60 years with male preponderance. Amongst malignant causes of ascites HCC (44.44\%), ovarian carcinoma $(33.33 \%)$, colon carcinoma $(11.11 \%)$, both gastric carcinoma and massive liver metastasis accounts for $5.55 \%$ each. Conclusion: The study highlights alcohol as the common culprit cause for ascites, labels HCC as the most common cause of malignant ascites, and defines the clinico-pathological, biochemical and imaging characteristics of different etiologies of ascites.
\end{abstract}

Key words: Ascites, Cirrhosis, Malignant, Metastasis, Alcoholic

\section{Introduction}

Ascites is the pathologic accumulation of fluid in the peritoneal cavity. It is one of the common presentations among patients admitted the tertiary care hospital amongst the many causes that can ultimately lead to ascites cirrhosis with Portal Hypertension is the commonest. Amongst the other causes of ascites metabolic diseases, cardiac diseases, malnutrition are important. Another pathology that is very important in Indian context is development of ascites from infections, where tuberculosis is most important. According to the

Manuscript received $18^{\text {th }}$ May 2016

Reviewed: $29^{\text {th }}$ May 2016

Author Corrected: $16^{\text {th }}$ June 2016

Accepted for Publication $30^{\text {th }}$ June 2016 first-ever World Health Organization, total deaths worldwide from cirrhosis and liver cancer rose by 50 million per year over 2 decades. In the year 2010, 1.3 million deaths worldwide are due to chronic viral hepatitis, which is comparable to the burden of HIV/AIDS, tuberculosis, and malaria [1].

Ascites due to malignancy deserves a special mention. Malignant ascites indicates the presence of malignant cells in the peritoneal cavity and is a grave prognostic sign. Survival in this patient population is poor, averaging about 20 weeks from time of diagnosis. Malignant ascites is a sign of peritoneal carcinomatosis 
(the presence of malignant cells in the peritoneal cavity). Tumours causing carcinomatosis are more commonly secondary peritoneal surface malignancies which include: ovarian, colorectal, pancreatic and uterine; extra-abdominal tumours originating from lymphoma, lung and breast; and a small number of unknown primary tumours. Studies have been conducted earlier with clinical, biochemical and imaging parameters associated with the different etiologies in patients with ascites [2]. In the present decade there is a notable change in the incidence of non-communicable as well as communicable diseases.

There is an increasing incidence of different Cancers in our country due to early detection, availability of investigation facilities and oncology services in government and private sectors. No recent study has been done in this part of the country to document the etiological pattern of ascites. This study is planned to throw some light on current picture of clinocopathological aspect of ascites with special reference to malignant etiology in this eastern part of India.

\section{Aim of the study}

The aim of this study is to explore the etiopathology, of ascites in the patients who were admitted to a tertiary care hospital in eastern India. The objectives of this present work is to study the distribution of etiology of ascites, alteration of blood and ascitic fluid parameters in patients with ascites, the imaging findings in patients admitted with ascites and the etiological causes of malignant ascites.

\section{Materials and Methods}

The study was conducted on 100 diagnosed patients of ascites in the in-patient department of General medicine, Medical College and Hospital, Kolkata for a period of one year from July 2014 to June 2015 after obtaining Institutional ethical clearance. Patients above $18 \mathrm{yrs}$ of age, diagnosed as Ascites confirmed by Ultrasound Abdomen were included in this Hospital based Cross-sectional study. Exclusion Criteria: Those who are already on treatment and/or have serious comorbid illness or Unable withstand invasive procedures have been excluded. No upper age limit has been set for our study. Complete Haemogram, Liver function test, Blood glucose (fasting / postprandial), Serum urea / creatinine, P-time, Ascitic fluid analysis, HBsAg, Anti $\mathrm{HCV}$ were performed. All the cases were studied as per the following Clinical- history, symptoms, clinical findings, Biochemical parameters - LFT and ascitic fluid study. The data were analyzed by SPSS 22.0.0.0 and tabulated using graphical representations.

Method of confirmation of malignant etiology: Ten milliliters of fresh peritoneal fluid sample was divided into two equal parts of five milliliters each. One part was subjected to the conventional smear cytology technique and the other part for the cell block technique. Thus, the same sample was evaluated for a comparative study.

The Conventional Smear Technique: The 5 milliliter sample was centrifuged at $2500 \mathrm{rpm}$ for 15 minutes. A minimum of 2 thin smears were prepared from the sediment. One smear was prepared after air drying and it was stained with the May-Grünwald-Giemsa stain. The other smear was immediately fixed in $95 \%$ alcohol and it was stained with the Papanicolaou stain.

The Cell Block Techniqu: The remaining $5 \mathrm{ml}$ sample was subjected to fixation for one hour by mixing it with $5 \mathrm{ml}$ of $10 \%$ alcohol-formalin (i.e., 9 parts of $90 \%$ alcohol and one part of $7.5 \%$ formalin). This $10 \mathrm{ml}$ fluid was centrifuged at $2500 \mathrm{rpm}$ for 15 minutes after one hour. A further $3 \mathrm{ml}$ of fresh $10 \%$ alcohol-formalin was once again added to the sediment after discarding the supernatant and it was kept for 24 hours. On the next day, the sediment which contained the cell button of the peritoneal fluid sample was scooped out on to a filter paper.

This cell button was processed along with other routine biopsy specimens. After paraffin embedding 4-6 $\mu$ thickness sections were prepared from this cell button and they were stained with the hematoxylin and eosin stain. Special stains like the Periodic Acid Schiff (PAS) and Mucicarmine were performed wherever they were necessary. After Confirmation of malignant etiology, Colonoscopy, Gastroscopy, CT guided FNAC, Pelvic USG with FNAC and CEA, CA 125 have been done to confirm primary etiologies.

\section{Results}

The majority of the subjects in our study group were males in all the group of patients, except in the NASH group where $50 \%$ were females and in the pancreatic ascites group where $100 \%$ were females. 
Table 1: Distribution of Etiology of Ascites.

\begin{tabular}{|c|c|c|}
\hline Etiology & No & Percentage \\
\hline Alcoholism & 36 & 36 \\
\hline Malignant Ascites & 18 & 17 \\
\hline HBV related ascites & 17 & 11 \\
\hline Tuberculosis & 11 & 8 \\
\hline HCV related ascites & 8 & 4 \\
\hline Wilsons Disease & 4 & 4 \\
\hline NASH & 4 & 1 \\
\hline Pancreatic ascites & 1 & 1 \\
\hline Budd-Chiari Syndrome & 1 & \\
\hline
\end{tabular}

As depicted in the above Table, the most common etiology is the alcoholism (36\%), followed by malignant ascites (18\%) and HBV related ascites (17\%). The other etiologies in the same order are tuberculosis $(11 \%)$, HCV related ascites $(8 \%)$, Wilson disease and NASH (both 4\%), pancreatic ascites and BCS (both 1\%).

Table 2: Distribution of Etiology of Malignant Ascites.

\begin{tabular}{|c|c|}
\hline Etiology & Percentage \\
\hline HCC & 44.44 \\
\hline Ovarian Ca & 33.33 \\
\hline Colon Carcinoma & 11.11 \\
\hline Gastric Ca & 5.55 \\
\hline Liver Metastasis & 5.55 \\
\hline
\end{tabular}

As depicted in the above diagram the most common etiology found amongst the cases of malignant ascites is HCC $(44.44 \%)$, followed in frequency by ovarian carcinoma $(33.33 \%)$ causing peritoneal carcinomatosis, colon carcinoma $(11.11 \%)$, and gastric carcinoma and massive liver metastasis (both $5.55 \%$ ).

Table-3: Comparison between malignant and non-malignant etiologies of ascites.

\begin{tabular}{|c|c|c|c|c|}
\hline & \multicolumn{2}{|c|}{ Etiology } & & \\
\hline & Non Malignancy & Malignancy & & \\
\hline & Mean \pm S.D & Mean \pm S D & P Value & Significance \\
\hline Platelet & $0.9 \pm 0.47$ & $1.59 \pm 0.91$ & $<0.001$ & Significant \\
\hline $\mathrm{MCV}$ & $91.62 \pm 17.76$ & $72.67 \pm 6.75$ & $<0.001$ & Significant \\
\hline PT INR & $1.74 \pm 0.45$ & $1.34 \pm 0.35$ & 0.001 & Significant \\
\hline ALP & $247.74 \pm 91.84$ & $375.78 \pm 340.39$ & 0.004 & Significant \\
\hline Ascitic fluid cell count & $229.13 \pm 195.06$ & $247.78 \pm 164.45$ & 0.707 & Not Significant \\
\hline Ascitic fluid pmn & $147.41 \pm 155.94$ & $82.5 \pm 86.37$ & 0.091 & Not Significant \\
\hline Ascitic fluid lymphocyte & $78.24 \pm 142.37$ & $73.33 \pm 103.18$ & 0.890 & Not Significant \\
\hline Ascitic fluid malignant cell & $0 \pm 0$ & $90.56 \pm 112.38$ & $<0.001$ & Significant \\
\hline Ascitic fluid protein & $2.06 \pm 0.36$ & $2.58 \pm 0.47$ & $<0.001$ & Significant \\
\hline Ascitic fluid albumin & $1.05 \pm 0.41$ & $1.66 \pm 0.58$ & $<0.001$ & Significant \\
\hline
\end{tabular}

Amongst alcoholic ascites 21 subjects (58.30\%) had hepatomegaly and 25 subjects $(69.40 \%)$ had splenomegaly. All of the HBV and HCV related ascites had splenomegaly (both 100\%), and none had hepatomegaly (both 0\%). In contrast all of the NASH subjects had hepatomegaly (100\%) none of them had splenomegaly. Regarding malignant ascites 11 had hepatomegaly $(61.1 \%)$ and 7 had splenomegaly (38.9\%). 5 of the tuberculous ascites $(45.5 \%)$ had splenomegaly but none had hepatomegaly. Wilson disease had 3 subjects with hepatomegaly and 3 with splenomegaly. Pancreatic ascites did not have hepatomegaly or splenomegaly. 1 BCS patient had both hepatomegaly and splenomegaly (both 100\%). All of the 
subjects of HBV related ascites $(\mathrm{n}=17 ; 100 \%)$ had HBsAg positivity; 5 malignant ascites subjects $(27.8 \%)$ and 1 BCS subject (100\%) had HBsAg positivity.

All of the subjects of HCV related ascites $(n=8 ; 100.0 \%)$ had Anti-HCV positivity and 3 subjects of malignant ascites (16.7\%) had Anti-HCV positivity. 4 subjects of alcoholic ascites (11.1\%), 2 of HBV related ascites (11.8\%), 1 of HCV related ascites (12.5\%), and 1 of tubercular ascites (9.1\%) had ICTC positivity.9 subjects with malignant ascites (50.0\%) and 1 with pancreatic ascites (100.0\%) ascitic fluid RBCs, that is hemorrhagic ascites. Amongst all the etiologies of ascites none of the subjects had smear positivity for any bacteria in ascitic fluid, irrespective of presence or absence of peritonitis. But, 5 subjects of alcoholic ascites (13.9\%), and 1 each of HBV and HCV related ascites (5.9\% and $12.5 \%$ respectively) had culture positivity for bacteria. All of the subjects, who had culture positivity, had spontaneous bacterial peritonitis.

USG were found to be abnormal in all subjects (100.0\%). Amongst the alcoholic ascites, the USG showed ascites and a liver with irregular edges and coarse echo-texture in majority of the patients. 21 subjects had hepatomegaly (58.3\%) and 25 had splenomegaly (69.4\%). Associated findings were splenomegaly, portosystemic collateral vessels, and reversal of the direction of flow in the portal vein (hepatofugal flow), with an increased portal vein diameter. HBV and HCV associated ascites subjects were shown to have abnormal USG findings in the form of ascites; shrunken liver with coarse echotexture; none of the subjects had hepatomegaly (both 100.0\%); and all of them had splenomegaly (both $100.0 \%$ ). Amongst the malignant ascites hepatomegaly were found in 11 subjects (61.1\%), and splenomegaly in 7 (38.9\%) along with ascites. Other USG findings varied as per the etiology of the malignancy. In HCC USG findings were nodules of different sizes; smaller tumours were uniformly hypoechoic, others were partly hyperechoic. All HCC patients had enhancement in the arterial phase (with the uninvolved liver lacking enhancement), loss of central nodule enhancement compared with the uninvolved liver (ishout), and capsular enhancement in the portal-venous and delayed phases in dynamic contrast-enhanced CT scan. In pancreatic ascites, USG showed large bulky pancreas; CT scan in addition showed contour irregularities and inhomogeneous attenuation with peripancreatic inflammation. UGIE did not reveal any abnormality. In the subjects with massive liver mets from colon carcinoma, USG and CECT scan showed multiple nodules in the liver suggestive of metastasis. CT scan in addition showed a growth in ascending colon suggestive of malignancy. UGIE showed portal hypertensive gastropathy (PHG). In ovarian carcinoma induced ascites USG and CT scan showed adnexal mass lesion arising from ovary along with ascites. UGIE failed to show any additional abnormality. In gastric carcinoma subject USG and CT scan showed large antral growth along with ascites; UGIE corroborated with the imaging findings. In colon carcinoma, USG and CT scan showed colonic growth and ascites; colonoscopy corroborated with the imaging findings; UGIE failed to show any abnormality. In tuberculous ascites, USG revealed septate ascites in all subjects $(\mathrm{n}=11,100.0 \%)$; none of them had hepatomegaly and 5 had splenomegaly (45.5\%). UGIE revealed duodenal ulcers in 8 subjects $(72.7 \%)$ majority of whom were alcoholic; along with PHG in two.

In Wilson disease, USG showed ascites with 3 having hepatomegaly and 1 splenomegaly. In BCS USG showed hepatic vein abnormalities, caudate lobe hypertrophy, increased reflectivity; Doppler ultrasound showed blood flow in the inferior vena cava and hepatic veins to be turbulent. CT scan showed enlargement of the liver with diffuse hypodensity before and patchy enhancement after contrast; along with heterogeneous hepatic parenchymal patterns. UGIE showed grade I to grade III varices, some of them had cherry-red spots, and hematocystic spots; portal hypertensive gastropathy (PHG) were also noted in majority.

\section{Discussion}

The most common etiology in our study is alcoholic liver disease $(36 \%)$, followed by malignant ascites $(18 \%)$ and $\mathrm{HBV}$ related ascites $(17 \%)$. The other etiologies in the same order are tuberculosis (11\%), $\mathrm{HCV}$ related ascites (8\%), Wilson disease and NASH (both 4\%), pancreatic ascites and BCS (both 1\%). If we club together the etiologies causing cirrhotic ascites, then that would make up $70 \%$ of the causations. This epidemiological result corroborates closely with other data which has been discribed by Hwanbo et al [3].
An Indian Study by Bhupinder Kumar, Brij Sharma et al [4] showed Cirrhosis of liver was the leading cause of ascites $(60.7 \%)$, tuberculosis was the second most common cause of ascites (13\%), malignancy and cardiac disorders were the third $(7.7 \%)$ most common causes for ascites. Alcohol was the leading cause of cirrhosis in 75 patients $(73.5 \%)$.

Another study from India by Bindu CB, Uday Nayak [5] showed Cirrhosis of liver was the most common 
cause of ascites (78\%) followed by Tubercular peritonitis $(8 \%)$ and alcohol was the commonest cause for cirrhosis of liver $(85 \%)$ followed by Hepatitis B virus infection.

We also documented the possible etiologies of malignant ascites. The data we collected showed the causative factors as: HCC (44.44\%), followed in frequency by ovarian carcinoma (33.33\%) causing peritoneal carcinomatosis, colon carcinoma $(11.11 \%)$, and gastric carcinoma and massive liver metastasis (both $5.55 \%$ ). When we divide this into two groups: peritoneal carcinomatosis group and $\mathrm{HCC} /$ massive liver metastasis group; we can see contribution from both groups are identical (49.99\%).

Now amongst peritoneal carcinomatosis most common is ovarian carcinoma $(66.66 \%)$ followed by colon $(22.22 \%)$ and gastric carcinoma $(11.11 \%)$. Other studies conducted on maalignant ascites have some very different data on it, as presented by Ayantunde AA et al [6] According to them ovarian carcinoma constitutes the largest contributor in etiology of malignant ascites with a percentage of $25 \%$. Other etiologies in decreasing frequency of causing malignant ascites were: breast cancer $(16 \%)$, gastric cancer $(13 \%)$, and colon cancer $(8 \%)$. The remainder were other etiologies $(8 \%)$ and carcinoma of unknown primary $(25 \%)$. This difference can be attributed to the small nature of sample size and different geographical region which might distort the clinical picture.

The age distribution of the cases did not show any statistically significant difference between the various group of patients. Sex distribution showed a higher predilection for the male sex.

Most of the subjects had pedal edema, except in tuberculosis etiology and in malignant etiology as mentioned in Sherlock' Diseases of Liver and biliary Systems [7]. Alcoholism is an important associated factor with ascites as shown in our study, where all the subjects with alcoholic liver diseases, had history of alcoholism (100\%); $41.2 \%$ of $\mathrm{HBV}$ related ascites, $62.5 \%$ of $\mathrm{HCV}$ related ascites and $63.6 \%$ subjects with tuberculous ascites had history of alcoholism. As regards to etiology, alcohol stands out as the leading cause of ascites in our study. In our study, amongst the alcohol related ascites group, 58.3\% had hepatomegaly, 69.4\% had splenomegaly, $33.3 \%$ had jaundice, Fever were documented in different etiologies of ascites; alcoholic ascites deserves here special mention; $47.2 \%$ of alcoholic ascites had fever. It had a mean MCV of $106.06 \mathrm{fL}, \mathrm{WBC}$ count of $7512.03 / \mathrm{mm}^{3}$, AST and ALT of $66.69 \mathrm{U} / \mathrm{L}$ and $51.92 \mathrm{U} / \mathrm{L}$, respectively, and serum albumin level of $2.62 \mathrm{~g} / \mathrm{dl}$. Other studies conducted on features of alcoholic liver diseases, as described by Mendenhall CL [8] corroborated to some extent with our study, where mean values of parameters in severe diseases were: MCV 105fL, WBC count 12000/ $\mathrm{mm}^{3}$, albumin $2.4 \mathrm{~g} / \mathrm{dl}$, AST and ALT of 99 and 57 respectively.

Next most frequent cause of ascites is malignancy, where we found cytology for malignant cell positive in 9 subjects $(50.0 \%)$ and negative in other 9 subjects $(50.0 \%)$ of malignancy associated ascites; along with it, none of the subjects had ascitic fluid albumin $>2.5 \mathrm{~g} / \mathrm{dl}$. Other studies on malignant ascites differs from us in this regard as described by Garrison $\mathrm{R} \mathrm{N}$ et al [9], where $57 \%$ had cytology for malignant cell positivity and $65 \%$ had ascitic fluid albumin level $>2.5 \mathrm{~g} / \mathrm{dl}$.

Here low platelet, ascitic fluid protein and ascitic fluid albumin; and high INR and MCV were significantly associated with non-malignant etiologies; whereas ascitic fluid cell counts, other than malignant cell, were not significant in distinguishing between malignant and non-malignant etiologies.This is in agreement with other studies [10].

Our study had some limitations. In our study we recruited diagnosed cases of ascites that might be on conservative treatment before enrolment. So, effect of previous treatment on our diagnostic workup and differential diagnosis were not taken into account. We have also excluded patients who are aged $<18$ years and elderly aged more than 65 years are less in our study. Ascites in the young, at one hand is an important clinical entity and Ascites in elderly on the other hand is an emerging national as well as global problem. So we did not bring into account the full spectrum of etiologies of ascites while analysing the causes which lead to ascites. Number of patients is 100 only in our study and duration is only one year. So this study is small and representative only.

\section{Conclusion}

From this hospital based cross-sectional study, we could conclude that, most common cause of ascites in tertiary hospital setting is alcohol related liver disease. So in community set up, we need to implement strategies to decrease the burden of alcoholism, which would in turn 
lead to decreased burden of alcoholic liver disease and alcohol related ascites. Also our study showed most common cause of malignant ascites to be HCC. So to decrease the burden of HCC in community setting we need to implement strategies.

Also our study shows importance of clinical, haematological, and biochemical parameters (for example history of alcoholism, hepatosplenomegaly, platelet, PT INR) in distinguishing malignant and nonmalignant etiologies and in establishing our provisional diagnosis.

Funding: Nil, Conflict of interest: None initiated.

Permission from IRB: Yes

\section{References}

\section{API Textbook of Medicine, Editors: Yash Pal Munjal.}

2. Philippe Langlet, Sylvie Escolano, Dominique Valla et al. Clinico-pathological forms and prognostic index in Budd-Chiari syndrome. Journal of Hepatology, Issue 4:496-499

3. Hwangbo Y. ET AL; Etiological and laboratory analysis of ascites in patients who underwent diagnostic paracentesis. Korean J Hepatol. 2007:13(2):185-195.

4. Bhupinder Kumar, Brij Sharma, Sujeet Raina, Neetu Sharma, Dalip Gupta, Kavita Mardi. Etiology of ascites in adults living in the Hills of Himachal Pradesh, India: A hospital-based study. Chrismed Journal of Medical Research. Year2016.Volume:3 Issue: 1: Page: 41-44.

5. Bindu CB, Uday Nayak. Clinical study of ascites with special reference to Serum-Ascitic Albumin Gradient. Int J Med Sci Public Health. 2014; 3(2): 169172.

6. Ayantunde AA, Parsons SL. Pattern and prognostic factors in patients with malignant ascites: a retrospective study. Ann Oncol 2007; 18(5): 945 - 9.

7. Sherlock's Diseases of the Liver and Biliary System, Twelfth Edition. Edited by James S. Dooley, Anna S.F. Lok, Andrew K. Burroughs, E. Jenny Heathcote. (C) 2011 by Blackwell Publishing Ltd. Published 2011 by Blackwell Publishing Ltd.

8. Mendenhall CL. Alcoholic hepatitis. Clin Gastro enterol, 1981 May;10(2):417-441.

9. Garrison R. N., Kaelin L. D., Galloway R. H., Heuser L.S. Malignant ascites: Clinical and experimental observation. Ann. Surg. 1986;203:644-644.

10. Kuiper JJ, van Buuren HR, de Man RA. Ascites in cirrhosis: a review of management and complications. Neth J Med. 2007 Sep. 65(8):283-8.

\section{How to cite this article?}

Ray D, Chakrabarti S, Chakraborty A, Kundu D, Bandyopadhyay R. A clinicopathological study of ascites with special reference to malignant etiology in a tertiary care hospital in Kolkata. Int J Med Res Rev 2016;4 (7):1102-1107.doi: 10.17511/ijmrr.2016.i07.05. 